YoKohama Mathematical

JOURNAL Vol. 40, 1992

\title{
TRIMMED SUMS OF MIXING TRIANGULAR ARRAYS WITH STATIONARY ROWS
}

\author{
$\mathrm{By}$ \\ Makoto MaEjima* and Yuko MoRita
}

(Received November 7, 1991)

\begin{abstract}
Limit laws of trimmed sums are studied for triangular arrays of rowwise stationary random variables. It is shown that if the marginal distribution of the array belongs to the domain of attraction of an infinitely divisible law without Gaussian component, the trimmed sum converges weakly to a nondegenerate random variable under some mixing and local dependence conditions.
\end{abstract}

\section{Introduction and results}

Let $\left\{X_{n j}, 1 \leqq j \leqq n ; n=1,2, \cdots\right\}$ be a triangular array of rowwise stationary real-valued random variables, where stationarity means that for any $1 \leqq i_{1}, \cdots$, $i_{k}, i_{1}+h, \cdots, i_{k}+h \leqq n,\left(X_{n, i_{1}}, \cdots, X_{n, i_{k}}\right)$ and $\left(X_{n, i_{1}+h}, \cdots, X_{n, i_{k}+h}\right)$ have the same distribution. For each $n$, let $X_{n}^{(k)}$ be the $k$-th largest among $\left\{X_{n 1}, \cdots, X_{n n}\right\}$ in the sense of absolute value such that $\left|X_{n}^{(1)}\right| \geqq \cdots \geqq\left|X_{n}^{(n)}\right|$. Let $S_{n}=\sum_{j=1}^{n} X_{n j}$ and define the modulus trimmed sum by

$$
\text { (r) } S_{n}=S_{n}-\sum_{k=1}^{r} X_{n}^{(k)},
$$

where $r \geqq 1$ is a fixed number of trimmed terms. In this paper, we shall study the asymptotic behaviour of ${ }^{(r)} S_{n}$.

There are two types of modulus trimming. In light trimming $r$ is fixed, and in moderate trimming $r=r_{n}$ depends on $n$ and in most cases $r_{n} \rightarrow \infty$ and $r_{n} / n$ $\rightarrow 0$ are assumed. Light trimming has been studied by [Dar], [AB], [H], [Ma] and [Mo]. Moderate trimming has been investigated by, e.g., [KL], [GP1] and [HK].

Natural order trimming is based on $X_{n 1}^{*} \leqq \cdots \leqq X_{n n}^{*}$, the order statistics of $\left\{X_{n 1}, \cdots, X_{n n}\right\}$, and the naturally trimmed sum is

$$
S_{n}\left(r_{n}, s_{n}\right)=S_{n}-\sum_{k=1}^{r_{n}} X_{n k}^{*}-\sum_{k=1}^{\delta_{n}} X_{n, n-k+1}^{*} .
$$

* Research completed during a visit to the Center for Stochastic Processes, University of North Carolina at Chapel Hill supported by the Air Force Office of Scientific Research Contract No. F49620 85C 0144.

1991 Mathematics Subject Classification : 60F05, 60E09.

Key words and phrases: trimmed sums, mixing random variables, infinitely divisible law, infinite series representation. 
${ }^{(2 r)} S_{n}$ and $S_{n}(r, r)$ are expected to have similar asymptotic behaviour, when symmetric around origin, but this is not necessarily true (cf. [CHaM] p. 695, [GP2] p. 1188). Natural trimming has been studied by, e.g., [CHoM], [GP1], [CHaM], [GP2]. We shall briefly mention this trimming at the end of the paper.

In all papers mentioned above, the random variables are i.i.d. Here we relax the condition of independence. Namely, our random variables are stationary, but not necessarily independent. However, we treat only triangular arrays whose marginal distributions belong to the domain of attraction of an infinitely divisible law without Gaussian component.

Our assumptions are as follows.

We first describe the assumptions (A.1) and (A.2) on the marginal distribution function. Let $F_{n}$ be the distribution function of $X_{n j}, 1 \leqq j \leqq n$, and let $\left\{\hat{X}_{n j}, 1 \leqq j \leqq n ; n=1,2, \cdots\right\}$ be the associated triangular array of $\left\{X_{n j}\right\}$ with independent random variables, namely for each $n,\left\{\hat{X}_{n j}\right\}$ are i.i.d. and $\hat{X}_{n j}$ has the distribution $F_{n}$.

(A.1) There exist $\left\{c_{n}\right\}$ such that $\sum_{j=1}^{n} \hat{X}_{n j}-c_{n}$ converges in law to an infinitely divisible random variable without Gaussian component whose characteristic function is given by

$$
f(\theta)=\exp \left\{\int_{R \backslash(0)} h(\theta, x) \boldsymbol{\nu}(d x)\right\},
$$

where $h(\theta, x)=e^{i \theta x}-1-i \theta x 1_{(|x| \leq 1)}$ and $\nu(\cdot)$ is a Lévy measure on $\boldsymbol{R} \backslash\{0\}$ satisfying $\int_{R \backslash(0)}\left(x^{2} \wedge 1\right) \nu(d x)<\infty$.

It is known (e.g., see [P]) that (A.1) is equivalent to the following two conditions :

$$
\left\{\begin{array}{rlrl}
n\left(1-F_{n}(x)\right) & \longrightarrow \nu((x, \infty)), & x>0, \\
n F_{n}(x) \longrightarrow \nu((-\infty, x]), & x<0,
\end{array}\right.
$$

at all continuity points $x$ of $\nu$, and

$$
\lim _{\varepsilon \rightarrow 0} \lim _{n \rightarrow \infty} n\left\{\int_{|x|<\varepsilon} x^{2} d F_{n}(x)-\left(\int_{|x|<\varepsilon} x d F_{n}(x)\right)^{2}\right\}=0 \text {. }
$$

(A.2) $\nu(\cdot)$ has a polar decomposition of product type such that for another Lévy measure $\rho(\cdot)$ on $(0, \infty)$ and for $0 \leqq p, q \leqq 1$ with $p+q=1$,

$$
\nu(A)=p \rho(A \cap(0, \infty))+q \rho((-A) \cap(0, \infty)), \quad A \in \mathscr{B}(\boldsymbol{R} \backslash\{0\}) .
$$

Hence, (1.2) can be written as

$$
\left\{\begin{aligned}
n\left(1-F_{n}(x)\right) & \longrightarrow p \rho((x, \infty)), & & x>0, \\
n F_{n}(-x) & \longrightarrow q \rho([x, \infty)), & & x>0 .
\end{aligned}\right.
$$


We next explain the assumptions (D.1), (D.2) and (D.3) on the joint distribution functions, namely some mixing, local dependence and other dependence conditions. Our conditions are those originally formulated by Leadbetter [Le] and then modified by Davis [Dav2], (also see [JK]).

(D.1) For every set $B$ which is a finite union of disjoint intervals of the form $\bigcup_{i=1}^{d}\left(a_{i}, b_{i}\right]$ whose closure does not contain 0 , as $n \rightarrow \infty$

$$
\sup \left|P\left\{X_{n j} \in B^{c}, p<j<r\right\}-P\left\{X_{n j} \in B^{c}, p<j<q\right\} P\left\{X_{n j} \in B^{c}, q<j<r\right\}\right| \rightarrow 0,
$$

where supremum is taken over all $p, q, r$ such that $0 \leqq p \leqq q \leqq r \leqq n$.

(D.2) For all $x>0$,

$$
\lim _{k \rightarrow \infty} \limsup _{n \rightarrow \infty} n \sum_{j=2}^{[n / k]} P\left\{\left|X_{n 1}\right|>x,\left|X_{n j}\right|>x\right\}=0 .
$$

$$
\lim _{\varepsilon \rightarrow 0} \limsup _{n \rightarrow \infty} n \sum_{j=2}^{n} \max \left\{0, \operatorname{Cov}\left(X_{n 1} 1_{\left(\left|X_{n 1}\right|<\varepsilon\right)}, X_{n j} 1_{\left(\left|X_{n},\right|<\varepsilon\right)}\right)\right\}=0 \text {. }
$$

To state our theorem, we need more notation. Let $\left\{\delta_{j}\right\}_{j=1}^{\infty}$ be a sequence of i.i.d. random variables such that $P\left\{\delta_{1}=1\right\}=p$ and $P\left\{\delta_{1}=-1\right\}=q$, where $p$ and $q$ are defined in assumption (A.2). Let $\left\{\Gamma_{j}\right\}_{j=1}^{\infty}$ be Poisson arrival times with unit rate, namely $\Gamma_{j}=e_{1}+\cdots+e_{j}$, where $\left\{e_{j}\right\}_{j=1}^{\infty}$ are i.i.d. random variables having the exponential distribution with mean 1 . We assume $\left\{\boldsymbol{\delta}_{j}\right\}$ and $\left\{\Gamma_{j}\right\}$ are independent of each other. Let $Y_{n k}=\left|X_{n}^{(k)}\right|$ and $\delta_{n k}=\operatorname{sign}\left(X_{n}^{(k)}\right)$ so that $X_{n}^{(k)}=\delta_{n k} Y_{n k}$ and ${ }^{(r)} S_{n}=\sum_{j=r+1}^{n} \delta_{n j} Y_{n j}$.

Our main theorems are the following:

Theorem 1. Assume (A.1), (A.2), (D.1), (D.2) and (D.3), and define

$$
R(u)=\inf \{x>0: \rho((x, \infty)) \leqq u\}, \quad u>0,
$$

as the right continuous inverse of the function $x \mapsto \rho((x, \infty))$. Then we have as $n \rightarrow \infty$,

where

$$
{ }^{(r)} S_{n}-{ }^{(r)} c_{n} \stackrel{w}{\longrightarrow}{ }^{(r)} Z
$$

$$
\begin{gathered}
{ }^{(r)} c_{n}=\sum_{j=r+1}^{n} E\left[\delta_{n j} Y_{n j} 1_{\left(Y_{n j} \leq 1\right\}}\right], \\
{ }^{(r)} Z=\sum_{j=r+1}^{\infty}\left\{\delta_{j} R\left(\Gamma_{j}\right)-(p-q) E\left[R\left(\Gamma_{j}\right) 1_{\left(R\left(\Gamma_{j}\right) \leq 1\right\}}\right]\right\},
\end{gathered}
$$

$\stackrel{w}{\rightarrow}$ denotes the weak convergence, and the infinite series in ${ }^{(r)} Z$ converges almost surely.

Theorem 2. The characteristic function of ${ }^{(r)} Z$ is given by 


$$
\begin{aligned}
E\left[e^{i \theta(r) z}\right]= & \frac{1}{(r-1) !} \int_{0}^{\rho((0, \infty))} u^{r-1} \exp \left\{-u+\int_{0<|x| \leq R(u)} h(\theta, x) \nu(d x)\right\} d u \\
& +\frac{1}{(r-1) !} \int_{\rho((0, \infty))}^{\infty} u^{r-1} e^{-u} d u
\end{aligned}
$$

Remark 1. Hall [H] studied the same problem for a sequence of independent random variables belonging to the domain of attraction of a non-normal stable law and obtained the limiting characteristic function. Later Mori [Mo] examined the limiting characteristic function of modulus trimmed sums when the law of the subsequential limit of sums of independent random variables is infinitely divisible. The limiting characteristic function in (1.5) is an extension of that in $[\mathrm{H}]$ where $\rho((x, \infty))=x^{-\alpha}, \rho((0, \infty))=\infty, R(u)=u^{-1 / \alpha}$, and also is the same as in Theorem 2 of [Mo].

\section{Preliminary lemmas (I)}

The proof of Theorem 1 can be carried out by the same idea as in Davis [Da2]. He applied the LePage representation (the infinite series representation) of stable random variables to prove the stable limit theorem for dependent random variables. Here we shall use the infinite series representation of infinitely divisible random variables recently developed by Rosinski [R].

Let $M_{n}^{k}$ and $W_{n}^{k}$ be, respectively, the $k$-th largest and smallest among $\left\{X_{n 1}, \cdots, X_{n n}\right\}$, and denote $\hat{M}_{n}^{k}$ and $\hat{W}_{n}^{k}$ for the associated i.i.d. sequence $\left\{\hat{X}_{n j}\right\}$ similarly. (Of course $M_{n}^{k}=X_{n, n-k+1}^{*}$ and $W_{n}^{k}=X_{n, k}^{*}$ by the notation in section 1.)

Lemma 2.1. ([Lo]) Under (1.2), as $n \rightarrow \infty$,

$$
\begin{aligned}
& P\left\{\hat{M}_{n}^{1} \leqq x\right\} \longrightarrow e^{-\nu([x, \infty))}, \quad x>0, \\
& P\left\{\hat{W}_{n}^{1}>x\right\} \longrightarrow e^{-\nu((-\infty, x])}, \quad x<0 .
\end{aligned}
$$

Lemma 2.2. Under (1.2), (D.1) and (D.2),

$$
\begin{aligned}
& P\left\{M_{n}^{1} \leqq x\right\} \longrightarrow e^{-\nu([x, \infty))}, \quad x>0, \\
& P\left\{W_{n}^{1}>x\right\} \longrightarrow e^{-\nu((-\infty, x])}, \quad x<0 .
\end{aligned}
$$

Proof. This is an analogue of Theorem 3.4.1 in [LLR] and it is enough to modify its proof slightly to the case of triangular arrays by using Lemma 2.1.

Lemma 2.3. Under (1.2), (D.1) and (D.2), the joint limiting distribution of two vectors 


$$
\left(M_{n}^{1}, \cdots, M_{n}^{k}\right) \text { and }\left(W_{n}^{1}, \cdots, W_{n}^{k}\right)
$$

is the same as that of

$$
\left(\hat{M}_{n}^{1}, \cdots, \hat{M}_{n}^{k}\right) \text { and }\left(\hat{W}_{n}^{1}, \cdots, \hat{W}_{n}^{k}\right)
$$

for each positive integer $k$.

Proof. The case where $\nu((-\infty, 0))>0$ and $\nu((0, \infty))>0$ is an analogue of Theorem 1 of Davis [Da2]. He proved the corresponding result in the case of single sequences by using Lemma 2.2 together with Theorem 4.2 and Remarks 1 and 2 in [Dal], which remain valid in our case of triangular arrays. Details are omitted.

The case where $\nu((-\infty, 0))=0$ or $\nu((0, \infty))=0$ is an analogue of Lemma 1 in $[\mathrm{Da} 2]$. We only prove the case $\nu((0, \infty))=0$. The case $\nu((-\infty, 0))=0$ is similar. It follows from $(1.2)$ that when $\nu((0, \infty))=0$, for any $\varepsilon>0$,

$$
n\left(1-F_{n}(\varepsilon)\right) \longrightarrow 0, \quad F_{n}(-\varepsilon) \longrightarrow 0 \quad \text { as } n \rightarrow \infty .
$$

Hence for $1 \leqq l \leqq k$ and for any $\varepsilon>0$,

$$
\begin{aligned}
P\left\{\left|M_{n}^{l}\right|>\varepsilon\right\} & \leqq P\left\{M_{n}^{k}<-\varepsilon\right\}+P\left\{M_{n}^{1}>\varepsilon\right\} \\
& \leqq P\left\{\bigcup_{j=1}^{k}\left\{X_{n j}<-\varepsilon\right\}\right\}+P\left\{\bigcup_{j=1}^{n}\left\{X_{n j}>\varepsilon\right\}\right\} \\
& \leqq k F_{n}(-\varepsilon)+n\left(1-F_{n}(\varepsilon)\right) \longrightarrow 0
\end{aligned}
$$

as $n \rightarrow \infty$. Thus, the vector $\left(M_{n}^{1}, \cdots, M_{n}^{k}\right)$ has a degenerate limit while $\left(W_{n}^{1}, \cdots\right.$, $\left.W_{n}^{k}\right)$ has the same limit as $\left(\hat{W}_{n}^{1}, \cdots, \hat{W}_{n}^{k}\right)$. This completes the proof.

We end this section with remarking the almost sure convergence of the infinite series in ${ }^{(r)} Z$. Recently, Rosinski [R] studied the infinite series representation of infinitely divisible random variables without Gaussian components. Our Lévy measure $\nu(\cdot)$ satisfying assumption (A.2) is a special case of those he treated in his paper. It follows from Corollary 4.8 of [R] that

$$
\sum_{j=1}^{n} \delta_{j} R\left(\Gamma_{j}\right)-(p-q) \int_{0}^{n} R(u) 1_{(R(u) \leq 1)} d u
$$

converges a.s. as $n \rightarrow \infty$ to an infinitely divisible random variable whose characteristic function is given by (1.1) with $\nu(d x)=p 1_{(x>0)} \rho(d x)+q 1_{(x<0)} \rho(d x)$. On the other hand, it is easy to see that as $n \rightarrow \infty$,

$$
\int_{0}^{n} R(u) 1_{(R(u) \leq 1)} d u=\sum_{j=1}^{n} E\left[R\left(\Gamma_{j}\right) 1_{\left(R\left(\Gamma_{j}\right) \leq 1\right)}\right]+o(1) .
$$

Hence

$$
\left.\sum_{j=1}^{\infty}\left\{\delta_{j} R\left(\Gamma_{j}\right)-(p-q) E\left[R\left(\Gamma_{j}\right)\right] 1_{\left(R\left(\Gamma_{j}\right) \leq 1\right)}\right]\right\}
$$


converges a.s., and so does ${ }^{(r)} Z$ for any $r \geqq 1$.

\section{Preliminary lemmas (II)}

Let $H_{n}(x)=P\left\{\left|X_{n j}\right|>x\right\}, x>0$. Then (1.4) implies

$$
n H_{n}(x) \longrightarrow \rho((x, \infty)), x>0, \quad \text { as } n \rightarrow \infty .
$$

Define $\hat{Y}_{n k}$ and $\hat{\delta}_{n k}$ for the associated i.i.d. sequence $\left\{\hat{X}_{n j}\right\}$ as $Y_{n k}$ and $\delta_{n k}$ for $\left\{X_{n j}\right\}$. We see that

$$
\left(\hat{Y}_{n 1}, \cdots, \hat{Y}_{n n}\right) \stackrel{d}{=}\left(H_{n}^{-1}\left(\Gamma_{1} / \Gamma_{n+1}\right), \cdots, H_{n}^{-1}\left(\Gamma_{n} / \Gamma_{n+1}\right)\right),
$$

where $H_{n}^{-1}(u)=\inf \left\{y: H_{n}(y) \leqq u\right\}$ and $\stackrel{d}{=}$ denotes the equality in distribution (see, e. g., Breiman [Br], Section 13.6).

Let us difine the following infinite-dimensional random vectors.

$$
\begin{gathered}
Z^{n}=\left(Y_{n 1}, \cdots, Y_{n n}, 0,0, \cdots\right) \in[0, \infty)^{\infty}, \\
\hat{Z}^{n}=\left(\hat{Y}_{n 1}, \cdots, \hat{Y}_{n n}, 0,0, \cdots\right) \in[0, \infty)^{\infty}, \\
Z=\left(R\left(\Gamma_{1}\right), R\left(\Gamma_{2}\right), \cdots\right) \in[0, \infty)^{\infty}, \\
\delta^{n}=\left(\delta_{n 1}, \cdots, \delta_{n n}, 1,1, \cdots\right) \in\{-1,1\}^{\infty}, \\
\hat{\delta}^{n}=\left(\hat{\delta}_{n 1}, \cdots, \hat{\delta}_{n n}, 1,1, \cdots\right) \in\{-1,1\}^{\infty}
\end{gathered}
$$

and

$$
\delta=\left(\delta_{1}, \delta_{2}, \cdots\right) \in\{-1,1\}^{\infty}
$$

Lemma 3.1. Under (1.4), $\hat{Z}^{n} \stackrel{w}{\rightarrow} Z$.

Proof. This is an analogue of Lemma 1 in [LWZ]. The same proof can be carried out by using (3.2).

Lemma 3.2. Under (1.4), (D.1) and (D.2), $Z^{n} \stackrel{w}{\rightarrow} Z$.

Proof. This is an analogue of Lemma 2 in [Dav2]. Under the assumptions for $\left\{X_{n j}\right\},\left\{\left|X_{n j}\right|\right\}$ satisfies the same assumptions. Thus we can apply Lemma 2.3 for $p=1$ to $\left\{\left|Y_{n j}\right|\right\}$ to conclude that $Z^{n}$ and $\hat{Z}^{n}$ have the same limiting distribution. The statement thus follows from Lemma 3.1.

Lemma 3.3. Under (1.4), $\hat{\delta}^{n} \stackrel{w}{\rightarrow} \delta$, and $\hat{\delta}^{n}$ is asymptotically independent of $\hat{Z}^{n}$.

Proof. This is an analogue of Lemma 2 in [LWZ] and the proof is quite similar, so we omit it. 
Lemma 3.4. Under (1.4), (D.1) and (D.2), $\delta^{n} \stackrel{w}{\rightarrow} \delta$, and $\delta^{n}$ is asymptotically independent of $Z^{n}$.

Proof. This is an analogue of Lemma 3 in [Dav2] and the same proof is carried out after replacing Theorem 1 in [Dav2] and Lemma 2 in [LWZ] by our Lemmas 2.3 and 3.3 above.

\section{Proofs of the theorems}

Proof of Theorem 1. The argument of the proof is the same as those in [LWZ] and [Dav2]. For notational convenience, let $[x ; A]=x 1_{A}(x)$ for an interval $A$. For $0<\varepsilon<\lambda$, let

and

$$
{ }^{(r)} S_{n}(\varepsilon, \lambda]=\sum_{j=r+1}^{n} \delta_{n j}\left[Y_{n j} ;(\varepsilon, \lambda]\right]
$$

Then

$$
{ }^{(r)} S(\varepsilon, \lambda]=\sum_{j=r+1}^{\infty} \delta_{j}\left[R\left(\Gamma_{j}\right) ;(\varepsilon, \lambda]\right]
$$

$$
{ }^{(r)} S_{n}-{ }^{(r)} c_{n}={ }^{(r)} S_{n}(0, \varepsilon]-E\left[{ }^{(r)} S_{n}(0, \varepsilon]\right]+{ }^{(r)} S_{n}(\varepsilon, \infty)-E\left[{ }^{(r)} S_{n}(\varepsilon, 1]\right] .
$$

To prove the theorem, it is enough to show (cf. Theorem 4.2 in [Bi]) that

$$
\begin{gathered}
\lim _{\varepsilon \rightarrow 0} \limsup _{n \rightarrow \infty} \operatorname{Var}\left[{ }^{(r)} S_{n}(0, \varepsilon]\right]=0, \\
{ }^{(r)} S_{n}(\varepsilon, \infty) \stackrel{w}{\longrightarrow}(r) S(\varepsilon, \infty) \quad \text { as } n \rightarrow \infty, \\
E\left[{ }^{(r)} S_{n}(\varepsilon, 1]\right] \longrightarrow E\left[{ }^{(r)} S(\varepsilon, 1]\right] \quad \text { as } n \rightarrow \infty
\end{gathered}
$$

and

$$
{ }^{(r)} S(\varepsilon, \infty)-E\left[{ }^{(r)} S(\varepsilon, 1]\right] \stackrel{w}{\longrightarrow}{ }^{(r)} Z \quad \text { as } \varepsilon \rightarrow 0 .
$$

To show (4.1), observe that

$$
\begin{aligned}
\operatorname{Var}\left[{ }^{(r)}\right. & \left.S_{n}(0, \varepsilon]\right]=\sum_{j=r+1}^{n} E\left[\left|\delta_{n j}\left[Y_{n j} ;(0, \varepsilon]\right]-E\left[\delta_{n j}\left[Y_{n j} ;(0, \varepsilon]\right]\right]\right|^{2}\right] \\
& +\sum_{j=r+1}^{n} \sum_{k=r+1, k \neq j}^{n} \operatorname{Cov}\left(\delta_{n j}\left[Y_{n j} ;(0, \varepsilon]\right], \delta_{n k}\left[Y_{n k} ;(0, \varepsilon]\right]\right) \\
\leqq & \sum_{j=1}^{n} E\left[\left|\delta_{n j}\left[Y_{n j} ;(0, \varepsilon]\right]-E\left[\delta_{n j}\left[Y_{n j} ;(0, \varepsilon]\right]\right]\right|^{2}\right] \\
& +\sum_{j=1}^{n} \sum_{k=1, k \neq j}^{n} \max \left\{0, \operatorname{Cov}\left(\delta_{n j}\left[Y_{n j} ;(0, \varepsilon]\right], \delta_{n k}\left[Y_{n k} ;(0, \varepsilon]\right]\right)\right\} \\
\leqq & n\left\{\int_{|x|<\varepsilon} x^{2} d F_{n}(x)-\left(\int_{|x|<\varepsilon} x d F_{n}(x)\right)^{2}\right\} \\
& +2 n \sum_{j=2}^{n} \max \left\{0, \operatorname{Cov}\left(\left[X_{n 1} ;[-\varepsilon, \varepsilon]\right],\left[X_{n j} ;[-\varepsilon, \varepsilon]\right]\right)\right\},
\end{aligned}
$$

where we have used the stationarity of $\left\{X_{n 1}, \cdots, X_{n n}\right\}$. We thus have (4.1) by (1.3) and (D.3). 
To show (4.2), define the subspace $W$ of $[0, \infty)^{\infty}$ consisting of all $w=$ $\left(w_{1}, w_{2}, \cdots\right)$ for which $w_{1} \geqq w_{2} \geqq \cdots \geqq 0$, and a function $\phi_{r}$ on $W \times\{-1,1\}^{\infty}$ by

$$
\phi_{r}(w, d)=\sum_{j=r+1}^{\infty} d_{j}\left[w_{j} ;(\varepsilon, \infty)\right]
$$

for $d=\left(d_{1}, d_{2}, \cdots\right) \in\{-1,1\}^{\infty}$ and $w=\left(w_{1}, w_{2}, \cdots\right) \in W$ for which $w_{j} \rightarrow 0$ as $j \rightarrow \infty$ and $\phi_{r}(w, d)=0$ for other values of $w$. Then as shown in [LWZ], $\phi_{r}$ is continuous in the product topology of $W \times\{-1,1\}^{\infty}$ at a.e. $(w, d)$ with respect to the distribution of $Z$ and $\delta$, and so by Lemmas 3.2 and 3.4 ,

$$
{ }^{(r)} S_{n}(\varepsilon, \infty)=\phi_{r}\left(Z^{n}, \delta^{n}\right) \stackrel{w}{\longrightarrow} \phi(Z, \delta)={ }^{(r)} S(\varepsilon, \infty)
$$

as $n \rightarrow \infty$ for $\varepsilon>0$, concluding (4.2). Note that the above proof also assures

$$
{ }^{(0)} S_{n}(\varepsilon, 1] \stackrel{w}{\longrightarrow}(0) S(\varepsilon, 1] \text {. }
$$

Also as in [LWZ], ${ }^{(0)} \hat{S}_{n}(\varepsilon, 1], n=1,2, \cdots$, are uniformly integrable, because $P\left(\left|\hat{X}_{n 1}\right|>\varepsilon\right) \sim n^{-1} \nu((\varepsilon, \infty))$, where $\hat{S}_{n}$ is defined for $\left\{\hat{X}_{n j}\right\}$. Then we have

$$
E\left[{ }^{(0)} S_{n}(\varepsilon, 1]\right]=E\left[{ }^{(0)} \hat{S}_{n}(\varepsilon, 1]\right] \longrightarrow E\left[{ }^{(0)} S(\varepsilon, 1]\right] \text {. }
$$

Therefore, to get (4.3) it suffices to show that

$$
E\left[\delta_{n j}\left[Y_{n j} ;(\varepsilon, 1]\right]\right] \longrightarrow E\left[\delta_{j}\left[R\left(\Gamma_{j}\right) ;(\varepsilon, 1]\right]\right]
$$

for $1 \leqq j \leqq r$. However, we see from Lemmas 3.2 and 3.4 that

$$
\delta_{n j}\left[Y_{n j} ;(\varepsilon, 1]\right] \stackrel{w}{\longrightarrow} \delta_{j}\left[R\left(\Gamma_{j}\right) ;(\varepsilon, 1]\right]
$$

and those random variables are bounded by 1 . The bounded convergence theorem concludes (4.5).

Finally, to show (4.4), note that

$$
\begin{aligned}
& { }^{(r)} Z-\left\{{ }^{(r)} S(\varepsilon, \infty)-E\left[{ }^{(r)} S(\varepsilon, 1]\right]\right\} \\
& \quad=\sum_{j=r+1}^{\infty}\left\{\delta_{j}\left[R\left(\Gamma_{j}\right) ;(0, \varepsilon]\right]-E\left[\delta_{j}\left[R\left(\Gamma_{j}\right) ;(0, \varepsilon]\right]\right\} .\right.
\end{aligned}
$$

Noting $\sum_{i=1}^{\infty} E\left[R\left(\Gamma_{j}\right)^{2}\right]<\infty$, we have that the variance of the above random variable is at most

$$
\sum_{j=1}^{\infty} E\left[\left[R\left(\Gamma_{j}\right) ;(0, \varepsilon]\right]^{2}\right] \leqq \sum_{j=1}^{\infty} \min \left\{\varepsilon^{2}, E\left[R\left(\Gamma_{j}\right)^{2}\right]\right\}
$$

which tends to zero as $\varepsilon \rightarrow 0$. This completes the proof of Theorem 1.

Remark 2. It may not be needed to assume (D.3), in particular, for the case when $\int_{0}^{\infty}(x \wedge 1) \rho(d x)<\infty$, but our proof requires it. 
Proof of Theorem 2. Although the form of the characteristic function of ( $r) Z$ can be given from Theorem 2 of [Mo], we give below a simpler proof. Note that

$$
\begin{aligned}
& { }^{(r)} Z=\sum_{j=r+1}^{\infty}\left\{\delta_{j} R\left(\Gamma_{j}\right)-(p-q) E\left[R\left(\Gamma_{j}\right) ;(0,1]\right]\right\} \\
& \stackrel{d}{=} \sum_{j=1}^{\infty}\left\{\delta_{j} R\left(\Gamma_{r}+\tilde{\Gamma}_{j}\right)-(p-q) E\left[R\left(\Gamma_{r}+\tilde{\Gamma}_{j}\right) ;(0,1]\right]\right\},
\end{aligned}
$$

where $\left\{\tilde{\Gamma}_{j}\right\}$ is an independent copy of $\left\{\Gamma_{j}\right\}$. It follows from Rosinski's result explained at the end of Section 2 that for each $u>0$, the random variable

$$
Z(u):=\sum_{j=1}^{\infty}\left\{\delta_{j} R\left(u+\tilde{\Gamma}_{j}\right)-(p-q) E\left[R\left(u+\tilde{\Gamma}_{j}\right) ;(0,1]\right]\right\}
$$

is infinitely divisible and has the characteristic function of the form (1.1) with the Lévy measure $\nu_{u}$ whose right continuous inverse is $R(u+\cdot)$, namely,

$$
\nu_{u}(d x)= \begin{cases}\nu(d x), & \text { if } u \leqq \rho((0, \infty)) \text { and }|x| \leqq R(u) \\ 0, & \text { otherwise. }\end{cases}
$$

Thus we can write

$$
E\left[e^{i \theta z(u)}\right]=\exp \left\{\int_{0<|x| \leq R(u)} h(\theta, x) \nu(d x)\right\},
$$

if we interpret $\int_{0<|x| \leq R(u)}$ to be zero when $u>\rho((0, \infty))$, equivalently, $R(u)=0$. Hence we conclude that ${ }^{(r)} Z$ is a mixture of infinitely divisible random variables and

$$
E\left[e^{i \theta^{(r)} z}\right]=\frac{1}{(r-1) !} \int_{0}^{\infty} u^{r-1} e^{-u} E\left[e^{i \theta Z(u)}\right] d u
$$

implying (1.5).

\section{Related results}

The argument in this paper up to here can be applied to prove other results related to extreme values. As an example, we show a result on natural trimming.

We have been assuming that the Lévy measure $\nu$ has a special polar decomposition (A.2). We do not need this restriction if we consider natural trimming based on the order statistics of $\left\{X_{n 1}, \cdots, X_{n n}\right\}$. Recall that $X_{n 1}^{*} \leqq \cdots$ $\leqq X_{n n}^{*}$, and set

We rewrite (1.1) as

$$
S_{n}(r, s)=\sum_{j=r+1}^{n-s} X_{n j}^{*}
$$

$$
f(\theta)=\exp \left\{\int_{-\infty}^{0-} h(\theta, x) d M(x)+\int_{0+}^{\infty} h(\theta, x) d N(x)\right\},
$$


where $M(x)=\nu((-\infty, x]), x<0$ and $N(x)=-\nu((x, \infty)), x>0$. Let for $u>0$,

and

$$
R_{1}(u)=\inf \{x<0 ; M(x)>u\}
$$

$$
R_{\mathbf{2}}(u)=\inf \{x>0 ;-N(x)>u\} .
$$

Let $\left\{\Gamma_{j}^{(1)}\right\}$ and $\left\{\Gamma_{j}^{(2)}\right\}$ be two independent copies of $\left\{\Gamma_{j}\right\}$. We have the following.

Theorem 3. Assume (A.1), (D.1), (D.2) and (D.3). Then we have

$$
\begin{aligned}
S_{n}(r, s)-\sum_{j=r+1}^{n-s} E[ & {\left.\left[X_{n j}^{*} ;[-1,1]\right]\right] } \\
\stackrel{w}{\longrightarrow} Z(r, s):= & \sum_{j=r+1}^{\infty}\left\{R_{1}\left(\Gamma_{j}^{(1)}\right)-E\left[\left[R_{1}\left(\Gamma_{j}^{(1)}\right) ;[-1,0)\right]\right]\right\} \\
& +\sum_{j=s+1}^{\infty}\left\{R_{2}\left(\Gamma_{j}^{(2)}\right)-E\left[\left[R_{2}\left(\Gamma_{j}^{(2)}\right) ;(0,1]\right]\right]\right\} .
\end{aligned}
$$

The characteristic function of $Z(r, s)$ is given by

$$
\begin{aligned}
E\left[e^{i \theta z(r, s)}\right]= & \frac{1}{\{(r-1) !\}^{2}}\left[\int_{0}^{M(0-)} u^{r-1} \exp \left\{-u+\int_{R_{1}(u)}^{0-} h(\theta, x) d M(x)\right\} d u\right. \\
& \left.+\int_{M(0-)}^{\infty} u^{r-1} e^{-u} d u\right] \\
& \times\left[\int_{0}^{-N(0+)} u^{r-1} \exp \left\{-u+\int_{0+}^{R_{2}(u)} h(\theta, x) d N(x)\right\} d u\right. \\
& \left.+\int_{-N(0+)}^{\infty} u^{r-1} e^{-u} d u\right] .
\end{aligned}
$$

Proof of Theorem 3. Some minor changes in the proof of Theorem 1 are enough to prove the first part of the theorem.

Instead of $Z^{n}, \delta^{n}$, etc. in Section 3 , we consider the following infinitedimensional random vectors.

$$
\begin{gathered}
Z_{(1)}^{n}=\left(\left[X_{n 1}^{*} ;(-\infty, 0)\right], \cdots,\left[X_{n, n-8}^{*} ;(-\infty, 0)\right], 0,0, \cdots\right) \in(-\infty, 0]^{\infty}, \\
\hat{Z}_{(1)}^{n}=\left(\left[\hat{X}_{n 1}^{*} ;(-\infty, 0)\right], \cdots,\left[\hat{X}_{n, n-8}^{*} ;(-\infty, 0)\right], 0,0, \cdots\right) \in(-\infty, 0]^{\infty}, \\
Z_{(1)}=\left(R_{1}\left(\Gamma_{1}^{(1)}\right), R_{1}\left(\Gamma_{2}^{(1)}\right), \cdots\right) \in(-\infty, 0]^{\infty}, \\
Z_{(2)}^{n}=\left(\left[X_{n n}^{*} ;(0, \infty)\right], \cdots,\left[X_{n, r+1}^{*} ;(0, \infty)\right], 0,0, \cdots\right) \in[0, \infty)^{\infty}, \\
\hat{Z}_{(2)}^{n}=\left(\left[\hat{X}_{n n}^{*} ;(0, \infty)\right], \cdots,\left[\hat{X}_{n, r+1}^{*} ;(0, \infty)\right], 0,0, \cdots\right) \in[0, \infty)^{\infty}, \\
Z_{(2)}=\left(R_{2}\left(\Gamma_{1}^{(2)}\right), R_{2}\left(\Gamma_{2}^{(2)}\right), \cdots\right) \in[0, \infty)^{\infty} .
\end{gathered}
$$

Lemma 5.1. ([J]) Under (A.1), 


$$
\hat{Z}_{(1)}^{n} \stackrel{w}{\longrightarrow} Z_{(1)} \text { and } \hat{Z}_{(2)}^{n} \stackrel{w}{\longrightarrow} Z_{(2)} \text {. }
$$

By Lemmas 2.3 and 5.1, we get

Lemma 5.2. Under (A.1), (D.1) and (D.2),

$$
Z_{(1)}^{n} \stackrel{w}{\longrightarrow} Z_{(1)} \text { and } Z_{(2)}^{n} \stackrel{w}{\longrightarrow} Z_{(2)} \text {. }
$$

To prove Theorem 3, as in the proof of Theorem 1, we have

$$
\begin{aligned}
S_{n}(r, s) & -\sum_{j=r+1}^{n-s} E\left[\left[X_{n j}^{*} ;[-1,1]\right]\right] \\
= & \sum_{j=r+1}^{n-s}\left(\left[X_{n j}^{*} ;[-\varepsilon, \varepsilon]\right]-E\left[\left[X_{n j}^{*} ;[-\varepsilon, \varepsilon]\right]\right]\right) \\
& +\sum_{j=r+1}^{n-s}\left(\left[X_{n j}^{*} ;(-\infty,-\varepsilon)\right]-E\left[\left[X_{n j}^{*} ;[-1,-\varepsilon)\right]\right]\right) \\
& +\sum_{j=r+1}^{n-s}\left(\left[X_{n j}^{*} ;(\varepsilon, \infty)\right]-E\left[\left[X_{n j}^{*} ;(\varepsilon, 1]\right]\right]\right)
\end{aligned}
$$

and need to show that

$$
\sum_{j=r+1}^{n-s}\left[X_{n j}^{*} ; A_{k}\right] \stackrel{w}{\longrightarrow} \sum_{j=\delta_{k}+1}^{\infty}\left[R_{k}\left(\Gamma_{j}^{(k)}\right) ; A_{k}\right], \quad k=1,2,
$$

where $A_{1}=(-\infty,-\varepsilon), A_{2}=(\varepsilon, \infty)$ and $\delta_{1}=r, \delta_{2}=s$,

$$
\sum_{j=r+1}^{n-s} E\left[\left[X_{n j}^{*} ; B_{k}\right]\right] \longrightarrow \sum_{j=\delta_{k}+1}^{*} E\left[\left[R_{k}\left(\Gamma_{j}^{(k)}\right) ; B_{k}\right]\right], \quad k=1,2,
$$

where $B_{1}=[-1,-\varepsilon), B_{2}=(\varepsilon, 1]$, and

$$
\begin{aligned}
& \sum_{j=\delta_{k}+1}^{\infty}\left\{\left[R_{k}\left(\Gamma_{j}^{(k)}\right) ; A_{k}\right]-E\left[\left[R_{k}\left(\Gamma_{j}^{(k)}\right) ; B_{k}\right]\right]\right\} \\
& \left.\quad \stackrel{w}{\longrightarrow} \sum_{j=\delta_{k}+1}^{\infty}\left\{R_{k}\left(\Gamma \xi^{(k)}\right)-E\left[\left[R_{k}(\Gamma\}^{(k)}\right) ;[-1,0)\right]\right]\right\},
\end{aligned}
$$

$k=1,2$, as $\varepsilon \rightarrow 0$.

(5.1) can be shown as in (4.1). To show (5.2) for $k=1$, define the subspace $W$ of $(-\infty, 0]^{\infty}$ consisting of all $w=\left(w_{1}, w_{2}, \cdots\right)$ for which $w_{1} \leqq w_{2} \leqq \cdots \leqq 0$, and a function $\phi_{r}$ on $W$ by

$$
\phi_{r}(w)=\sum_{j=r+1}^{\infty}\left[w_{j} ;(-\infty,-\varepsilon)\right]
$$

for $w \in W$ for which $w_{j} \rightarrow 0$ as $j \rightarrow \infty$ and $\phi_{r}(w)=0$ for other values of $w$. Then it is shown that $\phi_{r}$ is continuous in $W$ at a.e. $w$ with respect to the distribution of $Z_{(1)}$, and so by Lemma 5.2 ,

$$
\begin{aligned}
& \sum_{j=r+1}^{n-s}\left[X_{n j}^{*} ;(-\infty,-\varepsilon)\right]=\phi_{r}\left(Z_{(1)}^{n}\right) \\
& \quad \stackrel{w}{\longrightarrow} \phi_{r}\left(Z_{(1)}\right)=\sum_{j=r+1}^{\infty}\left[R_{1}\left(\Gamma_{j}^{(1)}\right) ;(-\infty,-\varepsilon)\right]
\end{aligned}
$$


as $n \rightarrow \infty$ for $\varepsilon>0$. A similar argument proves (5.2) for $k=2$. As to (5.3), we can show it by the same reasoning as in (4.3). Also, (5.4) is given by the same way as in (4.4), if we note that for $k=1,2$,

$$
\sum_{j=1}^{\infty} E\left[\left[R_{k}\left(\Gamma_{j}^{(k)}\right) ;[-\varepsilon, \varepsilon]\right]^{2}\right] \leqq \sum_{j=1}^{\infty} \min \left\{\varepsilon^{2}, E\left[R_{k}\left(\Gamma j_{j}^{(k)}\right)^{2}\right]\right\},
$$

where $\sum_{j=1}^{\infty} E\left[R_{k}\left(\Gamma_{j}^{(k)}\right)^{2}\right]<\infty$ because of the property of Lévy measures. This completes the proof of the first part of Theorem 3 .

The form of the characteristic function of $Z(r, s)$ is given by Theorem 2, if we regard $Z(r, s)$ as a sum of two independent infinitely divisible random variables, one of which is distributed as ${ }^{(r)} Z$ with $p=0$ and another is as ${ }^{(s)} Z$ with $p=1$ in Theorem 2 .

Acknowledgement The authors are very grateful to A. Janssen who sent us his unpublished manuscript.

\section{References}

[AB] D.Z. Arov and A.A. Bobrov, The extreme terms of a sample and their role in the sum of independent random variables, Theory Probab. Appl., 5 (1960), 377-396.

[Bi] P. Billingsley, Convergence of Probability Measures, Wiley, 1968.

[Br] L. Breiman, Probability, Addison-Wesley, 1968.

[CHaM] S. Csörgö, E. Haeusler and D. M. Mason, The asymptotic distribution of trimmed sums, Ann. Probab., 16 (1988), 672-699.

[CHoM] S. Csörgö, L. Horváth and D.M. Mason, What portion of the sample makes a partial sum asymptotically stable or normal? Probab. Th. Rel. Fields, 72 (1986), $1-16$.

[Dar] D. A. Darling, The influence of the maximum term in the addition of independent random variables, Trans. Amer. Math. Soc., 73 (1952), 95-107.

[Dav1] R.A. Davis, Limit laws for upper and lower extremes from stationary mixing sequences, J. Multivar. Anal., 13 (1983), 273-286.

[Dav2] R.A. Davis, Stable limits for partial sums of dependent random variables, Ann. Probab., 11 (1983), 262-269.

[GP1] P.S. Griffin and W.E. Pruitt, The central limit theorem for trimmed sums, Math. Proc. Camb. Phil. Soc., 102 (1987), 329-349.

[GP2] P.S. Griffin and W.E. Pruitt, Asymptotic normality and subsequential limits of trimmed sums, Ann. Probab., 17 (1989), 1186-1219.

[H] P. Hall, On the extreme terms of a sample from the domain of attraction of a stable law, J. London Math. Soc., (2) 18 (1978), 181-191.

[HK] M.G. Hahn and I. Kuelbs, Universal asymptotic normality for conditionally trimmed sums, Statist. Probab. Letters, 7 (1989), 9-15.

[J] A. Janssen, Sums of independent triangular arrays and extreme order statistics, Preprint, 1989.

[JK] A. Jakubowski and M. Kobus, $\alpha$-stable limit theorems for sums of dependent random vectors, J. Multivar. Anal., 29 (1989), 219-251.

[KL] J. Kuelbs and M. Ledoux, Extreme values and a Gaussian central limit theorem, Probab. Th. Rel. Fields, 74 (1986), 341-355. 
[Le] M.R. Leadbetter, On extreme values in stationary sequences, Z. Wahrsch. verw. Gebiete, 28 (1974), 289-303.

[LLR] M.R. Leadbetter, G. Lindgren and H. Rootzén, Extremes and Related Properties of Random Sequences and Processes, Springer, 1983.

[LWZ] R. LePage, M. Woodroofe and J. Zinn, Convergence to a stable distribution via order statistics, Ann. Probab., 9 (1981), 624-632.

[Lo] M. Loeve, Ranking limit theorem, Proc. Third Berkeley Symp. Math. Statist. Probab., 2 (1956), 177-194.

[Ma] R. Maller, Asymptotic normality of lightly trimmed means-a converse, Math. Proc. Camb. Phil. Soc., 92 (1982), 535-545.

[Mo] T. Mori, On the limit distributions of lightly trimmed sums, Math. Proc. Camb. Phil. Soc., 96 (1984), 507-516.

[P] V.V. Petrov, Sums of Independent Random Variables, Springer, 1975.

[R] J. Rosinski, On series representations of infinitely divisible random vectors, Ann. Probab., 18 (1990), 405-430.

Department of Mathematics,

Keio University

Hiyoshi, Yokohama 223, Japan

Current Address of

Yuko Morita

Yamaha Co.

Nishiyama-cho

Hamamatsu 432, Japan 